\title{
Precision grating for measuring microscope lens distortions
}

\author{
L. Jiang* \\ Electrical Engineering Department, Tuskegee University, Tuskegee, 36088 Alabama, USA
}

Received: 7 July 2010 / Accepted: 19 August 2010

\begin{abstract}
Microscopy is used in most technology processes where two-dimensional distributions, that are digital images of the shape and appearance of integrated circuit (IC) features, reveal important information. Optical microscopy and diffraction gratings can be used to measure the placement of IC features with a precision of less than $1 \mathrm{~nm}(1 \sigma)$ with a new technique of separately viewing multiple levels. However, the microscope's optical and video camera distortion may cause significant errors in the IC feature measurement under some circumstances. In this paper, the optical and video camera distortions of an optical microscope used in IC feature measurement were studied by analyzing digital images of a precision grating. MATLAB programs were used to extract the value of intensity of each pixel in the grating image. By matching a position dependent, phase-varied ideal sinusoidal wave to the processed grating digital image, the phase of the best-fit sine wave was observed to vary by the equivalent of many nanometers at different locations within the digital image of the grating. This variation was similar, but significantly different, for different microscope objectives. It is believed to arise from optical distortion within the microscope, and possibly also from distortion within the camera. Impact of optic chromatic aberration and microscope stability on the measurement of IC features was also studied. The method described here is inexpensive and easy to implement since it does not require any sophisticated equipment or controlled environment. This technique provides an attractive option for small companies, university labs and instrument manufacturers.
\end{abstract}

Keywords: Grating; lens distortion; integrated circuits metrology

\section{Introduction}

The smallest features in integrated circuits (ICs) currently in production are less than $45 \mathrm{~nm}$ wide, and their size is decreasing exponentially at the rate of about a factor of 2 every six years [1]. These features must be positioned at an accuracy of less than $10 \mathrm{~nm}$ [2].

Previous work $[3,4]$ has considered the measurement of placement of IC features within a level. The absolute position of features within a top pattern was measured by using a standard grid, or a grating, as the bottom pattern. The features were patterned in dyed resist on a transparent wafer. The dye [5] used was such that the resist had a high contrast at one wavelength, but was completely transparent at another. The transparent wafer was placed over a diffraction grating, and viewed by a microscope equipped with a video monochrome camera. By controlling the illumination, images were sequentially formed and captured of both the patterned level and the diffraction grating. Only the illumination was changed between image of the resist features and image of the grating. Measurements of the patterned level with respect to the grating provide an absolute measurement of the placement of its features, with an accuracy limited by the accuracy of the

^ Correspondence: ljiang@tuskegee.edu diffraction grating - state of art less than $2 \mathrm{~nm}, 3 \sigma[6,7]$. The absolute positional accuracy is required for measuring lithographic tool performance, e.g., distortions in an optical projection lens, or writing errors in an e-beam exposure tool.

Since distortion causes non-constant magnification, the measurement of distortion can be considered as measurement of magnification variation over the field. A standard approach is to use a calibration grid in the following manner: 1) capture image of grid with camera; 2) use machine vision software to find spots and calculate centroid of each; 3) transform data to remap [8]. In this work, a diffraction grating [9] was chosen for its advantages in measuring a microscope's optical distortion and video camera distortion. Recently, gratings have been fabricated with long range errors less than $2 \mathrm{~nm}, 3 \sigma$, and even more accurate gratings are expected in the future [7]. High precision gratings can be readily utilized in calibration. In addition, the centroid of a grating can be easily determined within thousandth of its period. A comprehensive analysis will be described in this paper on grating image position, microscope lens lateral chromatic aberration, microscope stability and their influence on the measurement of integrated circuit features. While measurements in only one direction are described here, the principle may be adapted to two dimensions. 
Filtered and normalized data

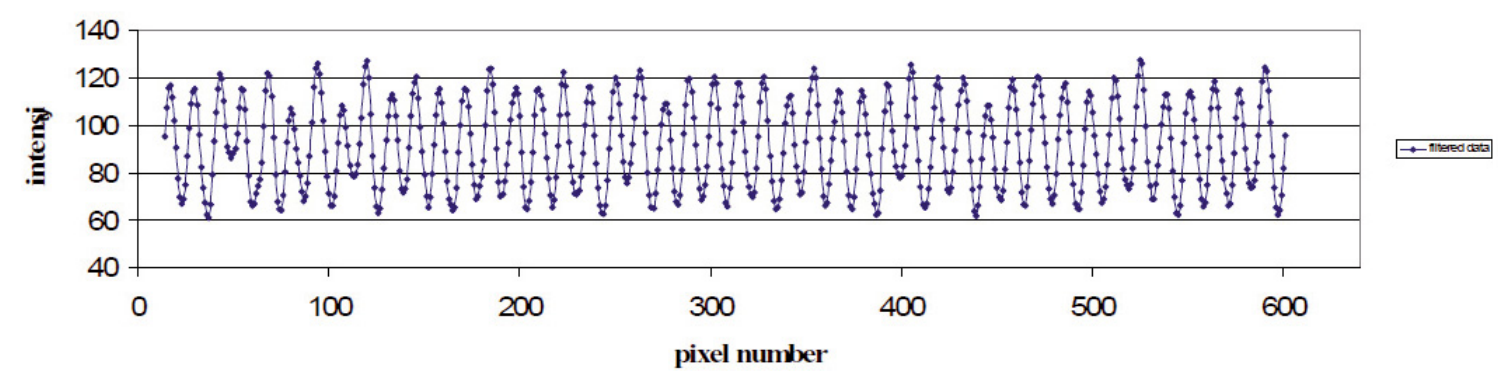

Fig. 1. A plot of the intensity distribution of the grating image versus pixels (in one direction) after filtering and normalization processing.

\section{Analysis of the microscope optics and camera distortions}

The grating image has both repeating and non-repeating components, and the repeating components may be represented by a Fourier series. The purpose of the analysis is to find the phase of the fundamental frequency in this Fourier series. A typical analysis of the grating image is as follows.

The grating was viewed under an Olympus microscope [10] equipped with monochrome CCD camera in transmitted HeNe laser coherent light or microscopeinclusive reflected incoherent light. The video image of the grating was taken by frame grabber software [11]. The digital image pixel resolution was $640 \times 480$. A MATLAB program extracted the value of intensity of each pixel in the digital image. The data consisted of a matrix of 640 columns and 480 rows and no sub-pixel interpolation was used. The field of view of the microscope was $53 \mu \mathrm{m}$ with a $100 \times$ objective, therefore approximately 13 pixels were involved in the resolving of one period of the periodic grating data by using $1.1 \mu \mathrm{m}$ period grating. Subsequently, the program averaged the data over all pixels in each column. The plot of the averaged data (ideally a sinusoidal wave) was irregular due to artifacts in the image. To eliminate the artifacts, both normalization and high pass filter processing were applied to the initial averaged data. Normalization corrects varying signal strength and high pass filtering removes slowly varying background level. The details of normalization and high pass filtering processing are described elsewhere [12]. One typical graph of the intensity distribution of the grating image versus pixels (in horizontal direction) is shown in Figure 1.

The processed video signal from the grating was subtracted from a generated ideal sine wave, and the phase of the ideal sine wave was adjusted to minimize the residuals through a MATLAB program. The phase corresponding to the minimum standard deviation of the residuals was defined as the phase of the grating image.

The units of the phase, standard deviation and error may be expressed in radians or nanometers. The conversion between the two units, for the $1.1 \mu \mathrm{m}$ period gratings: 1 radian $=1100 \mathrm{~nm} /(2 \pi)=175.07 \mathrm{~nm}$.
By matching a position dependent, phase-varied ideal sine wave to the processed grating video signal, it was possible to analyze the distortions of microscope optics and camera. The phase of the best-fit sine wave was observed to vary by the equivalent of many nanometers at different locations within the digital image of the grating. This variation was similar, but significantly different, for different microscope objectives. It is believed to arise from optical distortion of the microscope lenses, and possibly also from distortion within the camera. The variation in image position in the field of view with different wavelength of illumination was also analyzed for chromatic aberration in the optics.

Images of a $1.1 \mu \mathrm{m}$ period grating were taken under reflected incoherent illumination in the microscope. A thin layer of aluminum was deposited on top of the grating to enhance reflection and frames were taken with illumination of different wavelengths (blue, red by using interference filters and white) [13]. The aluminum deposition increased the contrast of the image significantly (Fig. 2). Consequently, the video signal of the aluminized grating image was much smoother than the ones without aluminum deposition. Nevertheless, the high pass filtering and normalizing processes were used to improve the data still further.

\subsection{Vertical variation in image position}

A vertical variation in image position was expected, since the grating could not be set exactly parallel to the vertical direction in the camera. To find the position variation in the vertical direction, the frame was divided into several parts from top to bottom and the best-fit phase value for each part was computed.

\subsubsection{Sample data}

The image positions in the vertical direction with illumination of different wavelength were compared (Fig. 3).

The image positions with:

Blue light $=74.86 \pm 25.68 \mathrm{~nm}$;

Red light $=66.23 \pm 25.29 \mathrm{~nm}$; 


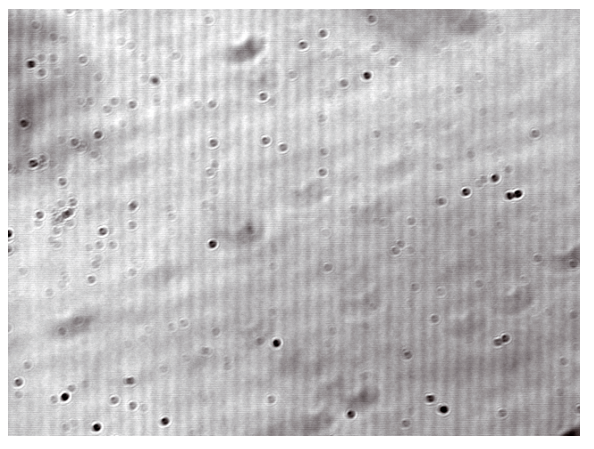

(a)

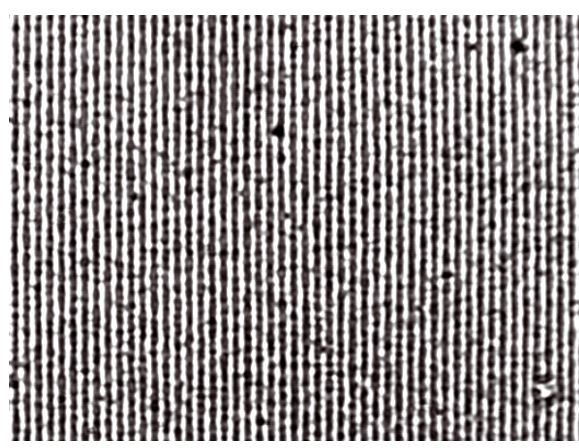

(b)

Fig. 2. Images of the grating under top reflected white light illumination (a) without aluminum coating and (b) with aluminum coating.

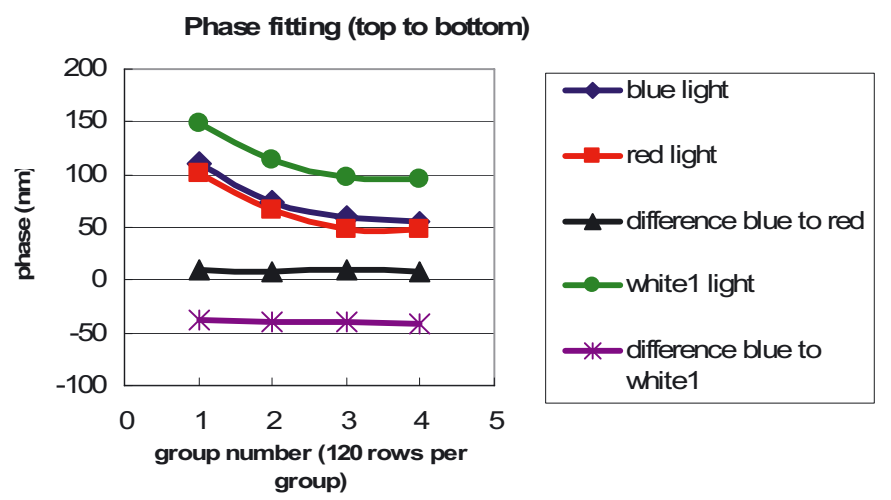

Fig. 3. Comparison of the image positions in vertical direction of the frame with different wavelengths.

White light $=114.13 \pm 24.79 \mathrm{~nm}$, where $\sigma$ is the standard deviation of the data.

Slope of the difference in image positions between:

Blue to red $=-2.3 \mathrm{~nm} / \mu \mathrm{m}$;

Blue to white $1=-5.2 \mathrm{~nm} / \mu \mathrm{m}$.

Where "white 1" refers to one of the digital images taken under white light illumination as indicated in Section 2.4.

\subsubsection{Discussion}

The deviation of the image positions was largely due to misalignment of the grating to the vertical direction of the camera. However, the fits are slightly curved, which shows there are more complex distortions. These will be measured in Section 2.2. The shape of the distortion from the top to the bottom of the frame was reproducible with different wavelengths. The difference of image positions with different wavelengths was likely caused by lateral chromatic aberration of the optics, which will be discussed in Section 2.3. The fact that the phase under white light did not fall between blue and red could arise from the microscope instability, which will be discussed in Section 2.4.

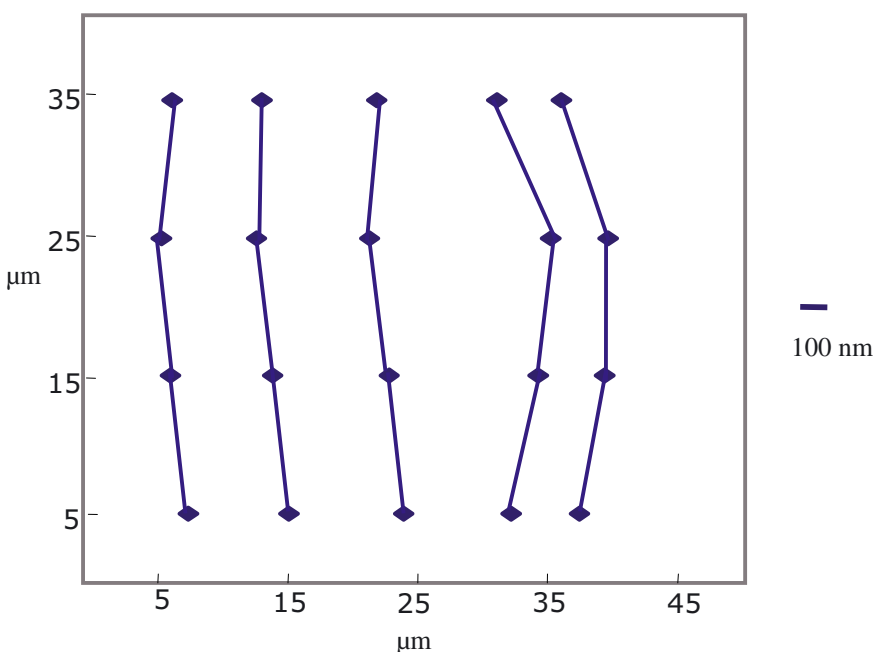

Fig. 4. The MARKET plot (matrix of 4 by 5 ) with blue light illumination. The scale bar at right measures the phase difference of each subfield with the units of phase expressed in nanometers.

\subsection{MARKET plot}

To map more complex distortions, the frame was evenly divided into 20 subfields (4 divisions in the vertical direction and 5 divisions in the horizontal direction). The image position in each section was measured and displayed in a MARKET plot (Fig. 4). MARKET plots are maps of two-dimensional displacements. The location of the grating in each subfield was calculated using the method in Section 2.

The plot in Figure 4 shows distortion only along the $x$ axis. In principle, the grating could be rotated 90 degrees and the process repeated to obtain distortion along the $y$ axis.

As shown in Figure 4, the phase of the best-fit sine wave varied at different locations within the digital image of the grating. To extract the maximum positional information from the entire field of view, the phase of the sine wave generated should vary across the field of view. The method used to generate a position dependent, 
Difference of Phase fitting (left to right)

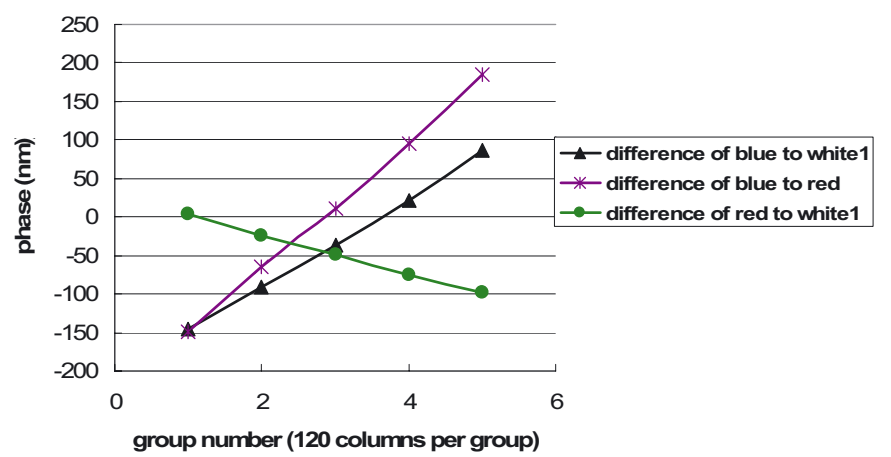

Fig. 5. Difference in distortion between different wavelengths.

phase varied sine wave was to fit a 4 th power polynomial $y=a+b x+c x^{2}+d x^{3}+e x^{4}$ to the phase values at each subfield. The variable $x$ of the polynomial is the column number and the function value $y$ is the corresponding phase value in radian.

It is important to note that for measuring distances between integrated circuit features, the distortion of microscope lens does not affect the measurement if the features are always centered in the same location of the field of view. The stage is intended to move from feature to feature, so they could all be measured in the same place. However, if the features are measured in different locations, appropriate compensation should be made for the optical/camera distortion.

\subsection{Chromatic aberration}

\subsubsection{Theory}

The index of refraction of glass varies as a function of wavelength, generally being higher for blue than for red and changes at a more rapid rate at shorter wavelenths [14]. An uncorrected single lens, therefore, has different focal lengths for different colors. Blue light comes to a focus closer to the lens than green or red light. A single lens is unable to bring all wavelengths of the spectrum into a common focus. This results in a slightly different focal point and image size for each predominant wavelength group [15]. An image formed in the blue focus is closer to the lens and smaller; at the red focus it is farther away and larger [16]. The horizontal distance between the two images is called longitudinal chromatic aberration. Achromatic lenses were introduced in microscopy to reduce longitudinal (axial) chromatic aberration. A lens doublet (also known as achromatic lenses) is formed by a combination of crown and flint lenses, where each lens has a different refractive index and dispersive properties. A doublet can reduce chromatic aberration by bringing two of the wavelength groups into a common focal plane with an optimal combination of lens thickness, curvature, refractive index, and dispersion. Modern microscopes utilize optical lens doublets or triplets made with three lens elements to reduce chromatic aberration [14].

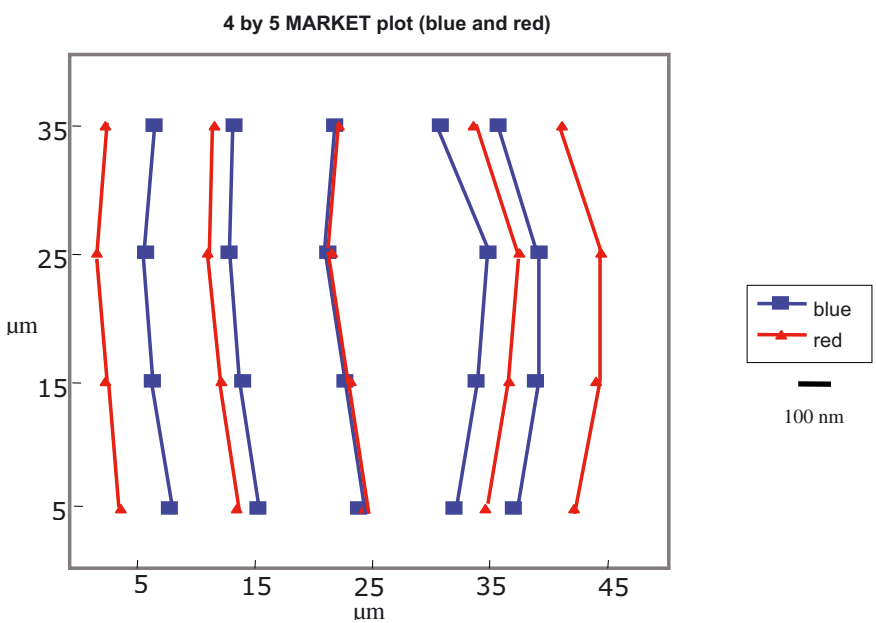

Fig. 6. MARKET plots (4 by 5 ) under blue light and red light illumination. The scale bar at right measures the phase difference of each subfield under blue light and red light illumination, with the units of phase expressed in nanometers.

In addition to longitudinal (or axial) chromatic aberration correction, microscope objectives also exhibit another chromatic defect. Despite bringing all three main colors to identical focal planes axially, such as in fluorite and apochromat objectives, the point images of details near the periphery of the field of view do not have the same size. This is called lateral chromatic aberration or chromatic difference of magnification [14]. A summary of aberrations, characters and corrections is provided by Meyer-Arendt in Table 5-1 of his book [16]. Although chromatic correction has been applied to the microscope optics, it must operate over the entire visible spectrum and is necessarily only approximate. The residual errors are large enough to produce significant effects.

\subsubsection{Measurement}

Since our technique of separately viewing multiple levels for measuring the placement of IC features requires measurements at different wavelengths, frames at the same location of the grating were compared at different wavelengths (Fig. 5). The effective wavelengths were 410$490 \mathrm{~nm}$ for the blue light and $560-640 \mathrm{~nm}$ for the red light. A linear plot implies a difference in magnification at the different wavelengths. The difference in magnification between red and blue light is also shown in a MARKET plot (Fig. 6).

In addition to the distortion errors already discussed, there are significant differences in magnification at different wavelengths. The images at different wavelengths are centered in the field of view. For the microscope used, the magnification was the largest in red light and the smallest in blue; and white light was intermediate. However, at the periphery of the field of view, lateral chromatic aberration is slightly greater for blue light (shorter wavelength). The effects are large compared to the error of the estimation, about $1.5 \mathrm{~nm}$, and cannot be ignored if more than 


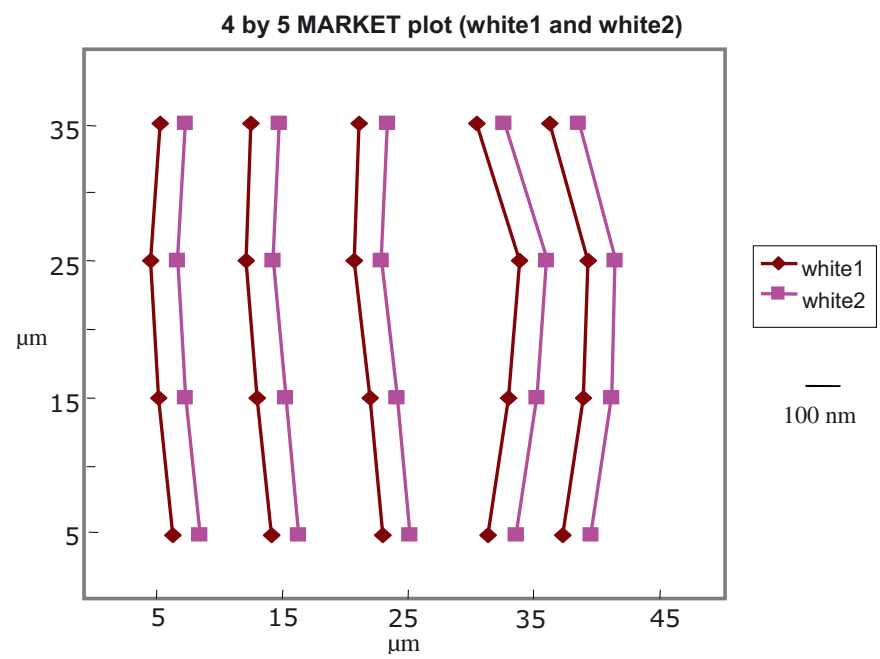

Fig. 7. MARKET plots ( 4 by 5 ) for two frames, taken a few seconds apart under white light illumination. The scale bar at right measures the phase difference of each subfield for both frames with the units of phase expressed in nanometers.

one wavelength is used, for example, to locate a feature viewed in blue light with respect to a grating viewed in red light. The amount of magnification error is approximately proportional to the distance between two features in the same field of view. However, the correction may be minimized by measuring all features at the same location in the field of view. But the features and gratings cannot be put in the same place for up to half the pitch of the gratings. The magnification error is about $200 \mathrm{~nm}$ across the field of view, which is $53 \mu \mathrm{m}$ with a $100 \times$ objective. If no correction is made within the half pitch distance, e.g. $0.55 \mu \mathrm{m}$, the error will be approximately $2 \mathrm{~nm}$. If the features are measured in exactly the same location of the field of view, the errors from distortion of the optics and camera are completely cancelled.

\subsection{Microscope stability}

Frames of the same location of the grating under white light were taken at intervals of a few seconds and compared. The stability of the microscope was found by computing how the sample moved with time.

The MARKET plot (Fig. 7) for two of the frames showed that the microscope moved in the transverse direction and no indication of rotation took place. It also shows that the distortion arising from the optics/camera is reproducible.

Microscope instability will not affect the measurement of distance between integrated circuit features if the features to be measured are in the same field of view.

Microscope instability will cause an offset in the measurement of distance between features if the features and gratings to be measured are in different fields of view by changing wavelengths of illumination (blue and red) sequentially. However, if the digital images of features and gratings are taken simultaneously with reflected blue illumination and transmitted red illumination by a color camera and there is no relative displacement between the features and the gratings, microscope instability does not matter.

\section{Conclusions}

Several sources of error contribute to the accuracy of measurement of microscope lens distortions. They are: (1) Location of the grating within the video field. Locating the grating is equivalent to finding the phase of a matching sine wave. This was easily determined to well below $1 \mathrm{~nm}$. (2) Errors in the grating. The characterization is limited by the accuracy of available gratings, and is readily upgraded by the substitution of more accurate gratings. Recently gratings have been fabricated with long range errors less than $2 \mathrm{~nm}, 3 \sigma$, and the prospect is that even more accurate gratings will be made in the future. (3) Thermal expansion of the grating. The temperature at which the lenses are measured should be close to the temperature at which the grating was patterned. However, the expected error from thermal expansion of grating is small compared to the scale of the microscope lens and camera distortion that was measured in this work.

For measuring distances between integrated circuit features, the distortion of microscope lens does not affect the measurement if the features are always centered in the same location of the field of view. However, if the features are measured in different locations, appropriate compensation should be made for the optical/camera distortion. The effects of microscope lens chromatic aberration cannot be ignored if more than one wavelength is used in measuring integrated circuits features, for example, to locate a feature viewed in blue light with respect to a grating viewed in red light. The amount of magnification error is approximately proportional to the distance between two features in the same field of view. Microscope instability will not affect the measurement of distance between integrated circuit features if the features to be measured are in the same field of view. Microscope instability will cause an offset in the measurement of distance between features if the features to be measured are in different fields of view.

Acknowledgements. The author thanks Professor Martin Feldman at the Department of Electrical and Computer Engineering at Louisiana State University (LSU) for his technical assistance and many helpful discussions. The author also thanks Mr. Golden Hwaung, Research Associate at the Electronic Material and Device Laboratory at LSU, for his assistance in metal deposition. This work was done at the Optical Laboratory at the Department of Electrical and Computer Engineering, LSU.

\section{References}

1. International Technology Roadmap for Semiconductors (ITRS), Executive Summary, http://www.itrs.net/Links/ 2009ITRS/Home2009.htm (2009)

2. ITRS 2008 update, http://www.itrs.net/Links/ 2008ITRS/Home2008.htm 
3. L. Jiang, M. Feldman, Portable coordinate measuring tool, J. Vac. Sci. Technol. B 23, 3056-3060 (2005)

4. L. Jiang, M. Feldman, Technique for separately viewing multiple levels, J. Vac. Sci. Technol. B 22, 3405-3408 (2004)

5. Coumarin dye number 314, Sigma-Aldrich Corporation, 3050 Spruce St., Saint Louis, MO 63103-2530

6. P.T. Konkola, C.G. Chen, R.K. Heilmann, C. Joo, J.C. Montoya, C.-H. Chang, M.L. Schattenburg, Nanometerlevel repeatable metrology using the Nanoruler, J. Vac. Sci. Technol. B. 21, 3097-3131 (2003)

7. M.L. Schattenburg, C. Chen, P.N. Everett, J. Ferrera, P. Konkola, H.I. Smith, Sub-100 nm metrology using interferometrically produced fiducials, J. Vac. Sci. Technol. B 17, 2692-2697 (1999)

8. www.optics.arizona.edu/ [Opti 415/515: Measurement of focal length and distortion by W.P. Kuhn] retrieved on 8 Sept. 2010

9. Holographic diffraction grating film catalog number R40267, Edmund Industrial Optics, 101 East Gloucester Pike, Barrington, NJ 08007-1380
10. Microscope model BX60, with objective model UPlanFl 1.30 NA, Olympus America Inc., 2 Corporate Center Drive Melville, NY 11747

11. INTELLICAM v2.05 software, Matrox Electronic Systems Ltd, boul. St-Régi Dorval, Québec, Canada H9P 2T4

12. L. Jiang, M. Feldman, A prototype optical encoder system with nanometer measurement capability, J. Mod. Optic. (2010) (in press)

13. Interference filter catalog number R46-156 and R46-159, Edmund Industrial Optics, 101 East Gloucester Pike, Barrington, NJ 08007-1380

14. http://www.microscopyu.com/tutorials/java/ aberrations/chromatic/index.html, retrieved on 8 Sept. 2010

15. http://www.olympusmicro.com/primer/lightandcolor/ opticalaberrations.html, [Common optical defects in lens systems (aberrations), Olympus Microscopy Resource Center] retrieved on 10 Sept. 2010

16. J.R. Meyer-Arendt, Introduction to classical and modern optics, 4th edn. (Prentice Hall, Upper Saddle River, New Jersey, 1995) 